\title{
USE OF DERMAPACE AND MEDIHONEY GEL AS A COMBINATION THERAPY IN THE TREATMENT OF COMPLICATED DIABETIC FOOT BURNS
}

Alejandra Monte Soldado, Jordi Serracanta Domènech, Mireia Ruiz Castilla

Plastic and Reconstructive Surgery Department of Vall d' Hebron Univesity Hospital, Barcelona, Spain.

OBJECTIVES: Surgical debridement of deep foot burns may expose tendon, bone or joint and can even lead to limbsacrificing surgery in diabetic patients.

We present our experience in the conservative management of diabetic foot burns with the combination of Medihoney wound gel and Dermapace in three adult patients.

PATIENTS AND METHODS: We present three cases of diabetic patients with complicated deep foot burns that were admitted in our burn unit between February and December 2016. The three patients had been treated in other centers for more than one week, and one of them had cellulitis signs. We used Dermapace twice a week (a medium of 9 sessions / patient) and dressings with Medihoney wound gel changed every 3 days.
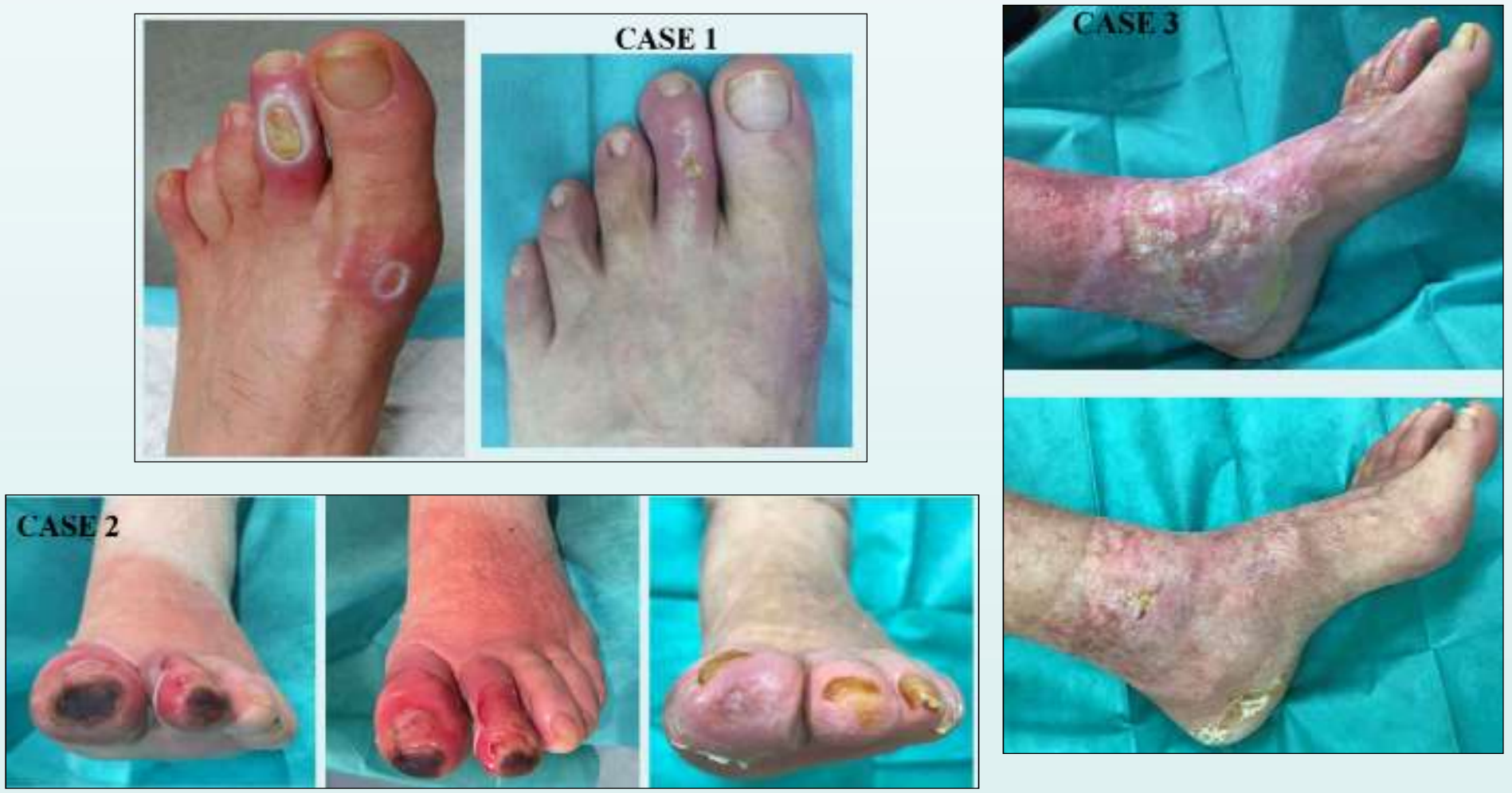

RESULTS: The three patients healed uneventfully within one and two months and all of them present a stable skin coverage and have returned to their daily activities

DISCUSSION: The peripheral neuropathy of diabetes mellitus leads to an increased frequency of foot burns. Due to the neurovascular changes associated, burns in these patients are usually deeper and difficult to heal; furthermore, they associate a high risk of infection and can lead to amputation of the extremity.

The clinical evidence in support of the effectiveness of honey in wound care has been reported by many authors ${ }^{1,2}$. It promotes debridement of necrotic tissue and stimulates granulation tissue formation by two mechanisms: high osmolarity and low $\mathrm{pH}$. Medihoney ${ }^{\circledR}$ wound gel is a patented formulation with Active Leptospermum Honey and natural gelling agents.

Pulsed acoustic cellular expression (PACE) technology has been developed based on scientific and clinical evidence of the beneficial wound healing effects of extracorporeal shock wave technology. PACE treatment stimulates angiogenesis and growth factor upregulation leading to the regeneration of tissue ${ }^{3}$.

CONCLUSION: In our experience, this combination therapy is useful in the conservative management of complicated foot burns in diabetic patients, avoiding more aggressive treatments in elderly, high-risk surgical patients.

\section{REFERENCES:}

1- Lusby PE, Coombes AL, Wilkinson JM 2005.Bactericidal Activity of different honeys against pathogenic bacteria. Arch Med Research; 36: 464-7

2- Blaser G et al 2007. Effect of medical honey on wounds colonised or infected with MRSA. J Wound Care. 16(8): 325-328

3-Cwykiel JM, Klimczak A, Krokowicz L, Siemionow M. Pre- and postischemic pulsed acoustic cellular expression conditioning modulates expression of inflammation factors in cremaster ischemia/reperfusion injury model. Microsurgery. $2013 \mathrm{Feb}$;33(2):134-40 